\title{
Cultura de la auditoría y la antropología en Colombia. Introducción a un debate urgente
}

\author{
Audit Culture and Anthropology in Colombia. \\ Introduction to an Urgent Debate
}

\author{
Santiago Martínez Medina* \\ Universidad de los Andes, Colombia
}



1 reciente XVI Congreso de Antropología en Colombia ${ }^{1}$ ofreció una serie de espacios centrales de debate cuyos argumentos aún resuenan en la cabeza de muchos. Gracias a la organización del evento, una de las discusiones giró en torno a un tema que, aunque no es usual en términos académicos, toca - en todos los sentidos de esa palabra- a los científicos sociales contemporáneos. Se trata de la manera en la que las políticas de la ciencia y la tecnología, basadas en la cultura de la auditoría, influyen, modifican, permiten y determinan nuestras propias prácticas intelectuales y académicas. Los ponentes invitados, los profesores Marta Zambrano y Yuri Jack Gómez-Morales, fueron los encargados de atizar, si se quiere, una discusión cuya pertinencia en el mundo científico contemporáneo no puede ni debe menospreciarse.

Médico de la Universidad Nacional de Colombia y doctor en Antropología de la Universidad de los Andes. Editor de Antípoda, la revista de antropología y arqueología de la Universidad de los Andes. Entre sus últimas publicaciones están: "Hacer arteria carótida en el laboratorio de anatomía. Práctica y materialidad en una asignatura de medicina" (2016), en Revista Colombiana de Sociología 39 (2): 31-47. Coautor con Julia Morales Fontanilla de "Entre muertos y especímenes: hacer cadáveres, anatomía y medicina legal en el laboratorio" (2016), en el Boletín de Antropología 30 (50): 127-147. s.martinez65@uniandes.edu.co.

1 El evento tuvo lugar en la Pontificia Universidad Javeriana de Bogotá, durante los días 6-9 de junio del 2017. 
De todos los debates centrales, "Políticas de la ciencia y la tecnología en Colombia” fue, por mucho, el que registró menos asistencia. Vale la pena reflexionar un momento sobre este fenómeno un tanto contradictorio. ¿Por qué si nos toca a todos parece que los debates en esta materia no fueran centrales en nuestra disciplina? Con gran tino, esa es precisamente una de las preguntas que los organizadores del evento les propusieron a los ponentes: "A pesar de las críticas planteadas, ¿por qué tales políticas terminan por ser apropiadas e instrumentalizadas?”. El texto que Yuri Jack Gómez-Morales publica en este número de la Revista Colombiana de Antropología apunta, particularmente, a resolver esa cuestión. Su respuesta, informada por su conocimiento crítico sobre la manera en la que la ciencia y la tecnología participan en la política, nos devuelve la pregunta a nosotros mismos. Si hoy por hoy nuestro trabajo se encuentra atenazado por prácticas de medición sobre las cuales muy poco reflexionamos, es porque nosotros mismos hemos participado en estas; las hemos autorizado ${ }^{2}$, en la medida en la que hemos hecho parte del juego que permite que la cultura de la auditoría (Strathern 2000) participe en el modo como hacemos ciencia social en el país. Es más, nos dice el profesor Gómez-Morales, la misma idea de que esas políticas son formuladas y ejecutadas por otros, diferentes a nosotros, es una "construcción ideológica” que sirve para librarnos de nuestra propia responsabilidad. Es también, a mi entender, una purificación (Latour 2007) que efectúa una separación a posteriori, con la capacidad de permitir que ciencia y sociedad se embrollen aún más, íntimamente, para producir auditoría como lo hacen. Tal vez por eso

Me inspiro aquí en Vinciane Despret (2008) cuando nos recuerda que el poder de la ciencia es la autoridad, para lo cual trae a colación a Gregory Bateson, quien define autoridad de la siguiente manera: "se dice que una persona tiene autoridad cuando alguien que está bajo su autoridad hace todo lo posible por hacer todo lo que esa persona dice ser cierto" $(2008,240)$. El debate que nos interesa no debe desestimar el hecho de que la cultura de la auditoría está montada sobre herramientas científico-técnicas sofisticadas, que rápidamente — tal vez con demasiada velocidad-, tendemos a considerar indiscutiblemente certeras. Nos parece un tanto obvio, por ejemplo, que un trabajo muy citado sea de calidad; de lo que se sigue que debamos medir nuestro índice $h$ (con Google Scholar o Publish or Perish), y solo cuando empezamos a ser medidos por aquello que nos resulta evidente, empezamos a sentir que algo no está bien. Esta sensación de cosa cierta es uno de los tantos temas que deberíamos pensar más, tal vez probando a ser idiotas, como lo propone Isabelle Stengers (2005), alzando la mano y preguntando sobre eso que a todo el mundo le parece tan obvio. Los efectos de este panorama tienen la capacidad de tocar no solo a la medición misma, sino también a nuestra propia experiencia ética sobre lo que hacemos. Marilyn Strathern se fija en la tríada ética, auditoría y política, y en la manera como cada una implica a las otras. Por eso, como lo señala el profesor Gómez-Morales, no debe sorprender que las instituciones estatales como Colciencias confundan en la política sobre la publicación científica auditoría y política, por ejemplo, cuando les fijan a las revistas científicas nacionales, como objetivo de fomento, que obtengan ciertos resultados en mediciones internacionales. En el mundo de la cultura de la auditoría, las fronteras en esa tríada son difusas. Por eso tampoco sorprende que obtener un mal resultado en esas mismas mediciones se vuelva paulatinamente sinónimo de no hacer bien la labor editorial. 
suponer que son otros los que hacen la medición nos sirve, no solo para dormir tranquilos, sino también para participar de la medición mientras hacemos el típico gesto de levantar los hombros y pensar que el asunto nos excede.

Las políticas de la medición terminan, entonces, por ser apropiadas e instrumentalizadas porque nosotros así lo autorizamos. El profesor Gómez-Morales vuelve el problema uno de nuestra propia participación, tan política como científica, en nuestro contexto de actuación. Como antropólogos no deberíamos estar sorprendidos ante esta conclusión. Reflexividad es, de hecho, una de las palabras claves de la antropología desde hace ya un buen tiempo. En los seminarios sobre etnografía se enseña que la persona y la situación del investigador hacen parte de la etnografía como medio y como fin. Se supone, por ende, que los antropólogos estamos más que capacitados para entender que la manera como hacemos nuestro trabajo participa de la creación del mundo que describimos. Tal vez, el punto que se nos escapa es que, en la medida en que hacemos nuestro trabajo, no solamente estamos creando el mundo sobre el cual hacemos etnografía, sino también el propio. Valdría, pues, la pena reflexionar tanto acerca de los efectos de la medición en nuestras prácticas, como de la forma en que nuestras prácticas participan y así autorizan la medición. Con toda seguridad, encontraremos que somos parte del problema tanto como las instituciones estatales con las que batallamos cada cierto tiempo ${ }^{3}$. ¿Qué estamos autorizando cuando nos negamos a participar como evaluadores de un artículo de alguna de las revistas nacionales?, ¿qué estamos autorizando cuando sometemos nuestros productos a diversos tipos de puntajes con o sin aspiración salarial?, ¿qué estamos autorizando cuando llegamos a la conclusión de que es siempre mejor publicar en inglés, como si el problema del abismo entre antropologías fuera solo una "cuestión de lenguaje"4? El debate en el pasado congreso nos recuerda que vale la pena hacerse preguntas sobre nuestras prácticas académicas más mundanas. Y es que en la práctica es donde hacemos la realidad, como lo argumentan los estudios de la ciencia y la tecnología, muchos de ellos - ya no debe sorprendernos- poniéndose en acción etnográficamente (Law 2004; Bonelli 2017).

La profesora Zambrano expuso la que puede ser una de las muchas maneras de responder a la cultura de la auditoría: volcar sobre nuestra propia academia

3 Batallamos no solo oponiéndonos, sino también quejándonos porque no son eficientes en la tarea de la que nos quejamos. ¿Cuántos de nosotros no nos hemos molestado porque la plataforma CvLAC o Publindex dejan de funcionar justo cuando vamos a diligenciar aquello que de tanto en tanto criticamos?

$4 \quad$ Parafraseo aquí el título de un artículo de Tania Pérez Bustos (2017) sobre la manera en la que nuestra condición inaudible frente a la academia en el norte global no es "solo una cuestión de lenguaje". 
las herramientas que hemos usado en el pasado para pensar antropológicamente tantos y tantos temas. Algo así como una suerte de mirada desde adentro y dentro de los mecanismos que hacen posible la medición. Una vez más, en un escenario tan complejo en el que no es fácil discernir los límites y las diferencias (y en el que cualquier producción de diferencia del tipo ellos los que miden, nosotros los medidos es tan fácil e improductiva), nos vemos abocados a pensar de formas novedosas - no euclidianas, podría decir inspirándome en Mol (2002)—, para imaginar que desde adentro podemos sacar los mecanismos mismos que hacen que la auditoría sea posible, para tal vez encontrar modos de subvertir una situación en la que "todo intento de insurrección termine sofocada...”, precisamente, desde adentro, como lo expone el profesor Gómez-Morales en su escrito.

A mi modo de ver, este es un reto no solo para nuestra capacidad analítica sino también para nuestra imaginación. Imaginación que debemos entender como un acto que no es exclusivo del pensamiento, sino que también consiste en abrir posibilidades; rutas de escape (Papadopoulus, Stephenson y Tsianos 2008), que como tales sean acciones también mundanas aunque potencialmente desestabilizadoras, maneras aún por figurarse precisamente porque no existen todavía. Por ende, no se trata de organizarse con timidez cada vez que llegan los momentos de la medición. El problema es bastante más complejo y excede el debate sobre la política de turno orientada a la medición de moda. El asunto es fundamentalmente el modo como hacemos el mundo con nuestra práctica académica y los límites que estamos dispuestos a guardar y a exceder en nuestros gestos más cotidianos. ¿Qué autorizamos cuando escribimos y evaluamos, también para las revistas colombianas? ¿Qué autorizamos cuando usamos como materiales pedagógicos documentos producidos en nuestras academias latinoamericanas? ¿Qué posibilidades estaríamos abriendo para nuestra disciplina si imaginamos mejores maneras de hablar entre nosotros? ¿Qué dejamos de autorizar cuando nos negamos a llamar a nuestro trabajo "producto"? Lo que está en tensión es la manera en la que hacemos academia leyendo, escribiendo, evaluando, enseñando, siendo interpelados e interpelando. La tarea es, pues, estar atentos para sentir la auditoría como interferencia en todas esas actividades y, así, un tanto tozudamente, preguntarnos si vale la pena hacer las cosas de otra forma. Si seguimos a los profesores Gómez-Morales y Zambrano, estas preguntas son algunas de las más urgentes en nuestra agenda científica y política hoy y en los años por venir. 


\section{Coda}

Por una feliz, aunque para nada azarosa, coincidencia, el pasado congreso albergó también una mesa de discusión entre los editores de las revistas colombianas de antropología y ciencias sociales. Motivados precisamente por las modificaciones a la política de publicaciones de ciencia y tecnología, nos reunimos para conversar sobre las implicaciones del nuevo sistema nacional de medición implementado por Publindex-Colciencias, situación que hace parte de la discusión sobre la cultura de la auditoría, a la cual nos hemos referido, y a nuestra responsabilidad y ética académica con las revistas nacionales. El mundo de la publicación científica, en un país como Colombia, demuestra la múltiple imbricación entre política, medición y ética a la que se refiere Strathern (2000), condimentada con una situación constante de precariedad que es ajena a la publicación en países como Estados Unidos o Inglaterra. Se trata, entonces, de sortear las tempestades cotidianas de políticas que confunden la medida con su objetivo, con equipos editoriales insuficientes, sin editores de tiempo completo en la mayoría de los casos, en ausencia de herramientas básicas de funcionamiento y con el escaso —sí, debe decirse - apoyo del gremio antropológico nacional. Los cambios en la política estatal para las revistas ponen en juego no solamente la manera como se mide su calidad, sino también su existencia misma como proyecto editorial. Si bien el encuentro fue solo un comienzo, concluimos que la colaboración es el modo de responder y de mejorar nuestra calidad, aunque no dudamos en hacer una serie importante de preguntas al gremio que hemos sentido ausente en el conflicto actual. ¿Nos leemos entre nosotros? ¿Usamos los materiales producidos en nuestra propia antropología? Nuestro propósito no es dar una respuesta a esas preguntas, sino invitar a todos los antropólogos a advertir una serie de transformaciones que, con toda seguridad, los tocan, de una forma u otra. Amanecerá y veremos, como dice el adagio popular. 


\section{Referencias}

Bonelli, Cristóbal. 2017. "Palabras de piedra, materiales proféticos y políticas del dónde”. Antípoda. Revista de Antropología y Arqueología 26: 19-43. DOI: http://dx.doi.org/10.7440/ antipoda26.2016.01.

Despret, Vinciane. 2008. "El cuerpo de nuestros desvelos. Figuras de la antropozoogénesis”. En Tecnogénesis: la construcción técnica de las ecologías humanas, vol. 1, compilado por Tomás Sánchez Criado, 229-261. Madrid: Asociación de Antropólogos Iberoamericanos en Red (AIBR).

Latour, Bruno. 2007. Nunca fuimos modernos. Ensayo de antropología simétrica. Madrid: Siglo XXI.

Law, John. 2004. After Method. Mess in Social Science Research. Londres y Nueva York: Routledge.

Mol, Annemarie. 2002. The Body Multiple: Ontology in Medical Practice. Londres: Duke University Press.

Papadopoulos, Dimitris, Niamh Stephenson y Vassilis Tsianos. 2008. Escape Routes. Control and Subversion in the Twenty-first Century. Londres: Pluto Press.

Pérez-Bustos, Tania. 2017. “'No es solo una cuestión de lenguaje’: lo inaudible de los estudios feministas latinoamericanos en el mundo académico anglosajón”. ScientleStudia São Paulo 15 (1): 59-72.

Stengers, Isabelle. 2005. "Introductory Notes on an Ecology of Practices”. Cultural Studies Review 11 (1): 183-196.

Strathern, Marilyn. 2000. “Afterword: Accountability and Ethnography”. En Audit Cultures. Anthropological Studies in Accountability, Ethics and the Academy, 279-304. Londres y Nueva York: Routledge. 